\title{
Impacto na saúde mental do paciente com acne grave/moderada em uso da isotretinoína oral: Estudo qualitativo
}

Impact on the mental health of severe/moderate acne patients taking oral isotretinoin: A qualitative study

Impacto en la salud mental de los pacientes con acné grave/moderado que utilizan isotretinoína oral: Un estudio cualitativo

Karoline Michaely Nóbrega Saraiva ORCID: https://orcid.org/0000-0001-8251-3608 Centro Universitário de Patos, Brasil E-mail: karolinemns@gmail.com Lívio Vasconcelos do Egypto ORCID: https://orcid.org/0000-0001-7804-4116 Centro Universitário de Patos, Brasil E-mail: livioegypto@gmail.com

\begin{abstract}
Resumo
A acne grave/moderada é uma doença genético-hormonal autolimitada que se relacionada ao processo inflamatório do aparelho pilossebáceo, levando à formação de abscessos (nódulos purulentos) e cistos intercomunicantes, além de possíveis alterações sistêmicas. O prognóstico é variável; quanto mais cedo for feito o tratamento correto com o uso da Isotretinoína oral, e forem seguidas as devidas instruções do médico dermatologista, melhor o resultado do paciente. Objetivo: Analisar a existência de correlação do uso da Isotretinoína oral em pacientes com acne grave/moderada, com um impacto negativo na sua saúde mental. Metodologia: Análise de casos que tem como característica ser um estudo observacional de caráter prospectivo e apresenta abordagem qualitativa. As informações foram obtidas por meio da realização de questionários e da coleta de dados dos prontuários dos pacientes, isso, só pôde ser realizado após autorização por escrito mediante assinatura do Termo de Consentimento Livre e Esclarecido (TCLE) e o consentimento do Comitê de Ética em Pesquisa do Centro Universitário de Patos (UNIFIP). Resultados: $\mathrm{O}$ estudo proposto obteve um resultado positivo quanto aos efeitos do medicamento no tratamento da acne grave/moderada, bem como na saúde mental dos pacientes. Conclusão: Dessa forma, pode contribuir significativamente com o melhor bem estar dos pacientes em tratamento, assim como com uma melhor conduta por parte dos profissionais ao se tratar de um cuidado maior com os efeitos psíquicos relacionados ao medicamento.
\end{abstract}

Palavras-chave: Isotretinoína; Acne; Saúde mental.

\begin{abstract}
Acne severe/moderate is a self-limited genetic-hormonal disease that is related to the inflammatory process of the pilosebaceous apparatus, leading to the formation of abscesses (purulent nodules) and intercommunicating cysts, besides possible systemic alterations. The prognosis is variable; the sooner the correct treatment with oral isotretinoin is done, and the instructions of the dermatologist are followed, the better the outcome for the patient. Objective: To analyze whether there is a correlation between the use of oral Isotretinoin in patients with severe/moderate acne, and a negative impact on their mental health. Methodology: Case analysis that has the characteristic of being an observational study of prospective character and presents a qualitative approach. The information was obtained by means of questionnaires and data collection from the patients' medical records, which could only be done after written authorization by signing the Informed Consent Form (ICF) and the consent of the Research Ethics Committee of the Patos University Center (UNIFIP). Results: The proposed study obtained a positive result regarding the effects of the medication in the treatment of severe/moderate acne, as well as on the mental health of the patients. Conclusion: Thus, it may contribute significantly to the better well being of patients in treatment, as well as to a better conduct on the part of professionals when it comes to greater care with the psychic effects related to the medication.
\end{abstract}

Keywords: Isotretinoin; Acne; Mental health.

\section{Resumen}

El acné gravis/moderado es una enfermedad genético-hormonal autolimitada que está relacionada con el proceso inflamatorio del aparato pilosebáceo, dando lugar a la formación de abscesos (nódulos purulentos) y quistes intercomunicados, además de posibles alteraciones sistémicas. El pronóstico es variable; cuanto antes se realice el 
tratamiento correcto con isotretinoína oral y se sigan las instrucciones del dermatólogo, mejor será el resultado del paciente. Objetivo: Analizar la existencia de una correlación entre el uso de Isotretinoína oral en pacientes con acné severo/moderado, con un impacto negativo en su salud mental. Metodología: Análisis de casos que tiene la característica de ser un estudio observacional de carácter prospectivo y presenta un enfoque cualitativo. La información se obtuvo a través de cuestionarios y de la recogida de datos de las historias clínicas de los pacientes, que sólo se pudo realizar tras la autorización escrita mediante la firma del Formulario de Consentimiento Informado (FCI) y el consentimiento del Comité de Ética de la Investigación del Centro Universitario de Patos (UNIFIP). Resultados: El estudio propuesto obtuvo un resultado positivo en cuanto a los efectos de la medicación en el tratamiento del acné severo/moderado, así como en la salud mental de los pacientes. Conclusión: Así, puede contribuir significativamente a la mejora del bienestar de los pacientes en tratamiento, así como a una mejor conducta por parte de los profesionales a la hora de tener un mayor cuidado con los efectos psíquicos relacionados con la medicación.

Palabras clave: Isotretinoína; Acné; Salud mental.

\section{Introdução}

A acne vulgar é uma doença comum multifatorial, mais frequente em $80 \%$ na população mais jovem, surgindo na puberdade e podendo permanecer até a idade adulta (Araújo, Delgado \& Marçal, 2011). Trata-se de uma condição patológica genética-hormonal e autolimitada, que ocorre devido à inflamação dos folículos pilossebáceos e consequente evolução para abcessos e pústulas, ou cistos intercomunicantes. Pode-se ainda definir a acne vulgar como uma dermatose crônica, sendo essa de equivalente acometimento para ambos os sexos (Abreu, Martins, Uchôa, Falcão \& Bezerra, 2013).

A acne por si só, muitas vezes já é considerada como uma doença psicodermatológica, ou seja, não afeta somente a pele, mas também causa danos e transtornos emocionais. Esse fato evidencia a necessidade de busca por psicoterapia ou tratamento psicoterápico na tentativa de contribuir também para uma melhora dermatológica (Isidorio et al., 2020).

Tratando-se de espectro clínico, a acne pode variar de comedonal (grau I), evoluindo para pápulas e pústulas (graus II e III), até lesões nóduloscísticas, que caracterizam o grau de inflamação mais avançado e prejudicial ao paciente, chegando a desencadear um acometimento sistêmico: acne fulminans, ou de grau IV (Sampaio \& Bagatin, 2008). De forma sucinta, a etiopatogenia dessa condição inicia-se com o aumento da secreção das glândulas sebáceas, diretamente influenciada por ação hormonal, levando à obliteração das mesmas juntamente com a retenção de sebo. Formam-se assim, os comedões. O processo de retenção ocasiona a proliferação de microrganismos, sobretudo o anaeróbio propioniobacterium, que atua liberando ácidos graxos livres na pele, encadeando o potencial inflamatório da acne (Sampaio \& Rivitti, 2014).

Para os casos mais leves, o tratamento clínico e farmacológico da patologia é baseado no uso tópico de sabonetes desengordurantes, com ácido salicílico e ácido retinóico, até no máximo um ciclo de antibiótico sistêmico, somados a dietas mais saudáveis e cuidados prévios com a proteção solar. No entanto, para as inflamações mais intensas, em que a acne é grave, nódulo-cística e conglobata, ou ainda, resistente a outras formas de tratamento, o mais indicado é o medicamento retinóide de ação antisseborréica Isotretinoína, comercialmente denominado de Roacutan (Azulay, 2013).

Sabe-se que atualmente, a cepa do Propionibacterium acnes, o agente infeccioso responsável pela presença e manifestação da acne vulgar, possui um crescente mecanismo de resistência a antibióticos, o que dificulta o tratamento medicamentoso (Lima, Barros \& Neto, 2020). No entanto, existem alternativas para casos em que isso acontece; como a utilização da medicina alternativa, por exemplo, que têm como instrumento principal plantas medicinais e seus compostos bioativos, atuantes nas mais diversas vias fisiopatológicas da acne (Cubas \& Ribas, 2021). Ademais, constata-se que há uma certa eficácia na utilização de óleos essenciais e extratos vegetais como alternativa para tratamento, já que esses apresentaram algum efeito antibiótico, antimicrobiano, sinérgico e até anti-inflamatório (Miranda, 2020).

Outra técnica que vem se expandindo e encontra-se bastante difundida é o microagulhamento, que utiliza da indução percutânea de colágeno para tratar cicatrizes causadas pela acne (Costa, Souza, Conradesque, Carniel \& Zago, 2020). De mesmo modo, há quem defenda a necessidade de múltiplas terapêuticas seja por meio de retinóides, corticóides, 
anticoncepcionais orais, peróxido de benzoíla, seja pela formulação de extratos advindos da natureza, intercalando entre tópicos e sistêmicos, para uma maior eficácia em tratamento (Barbosa et al., 2021).

A Isotretinoína oral, no entanto, é segundo a opinião de especialistas dermatológicos, o que de mais eficaz o mercado farmacológico oferta atualmente para tratar acne e possui em sua fórmula uma substância derivada da vitamina A, causando uma melhora clínica associada à redução do tamanho e da atividade das glândulas sebáceas. Além disso, o resultado clínico estimado varia de 8 a 16 semanas (Vinhal, Roberth, Ortence \& Diniz., 2014).

O cuidado continuado com o acompanhamento clínico do tratamento (são necessários exames laboratoriais mensais), aliado ao fato de ser teratogênico e trazer em sua bula um razoável leque de efeitos colaterais, costumam assustar os pacientes que precisam fazer uso do mesmo e a população em geral, que tanto dissemina más informações. Vale ressaltar, no entanto, que os efeitos subsequentes ao consumo do medicamento, são em sua grande maioria irrelevantes quando comparados aos benefícios decorrentes do seu uso. Os efeitos adversos associados a todos os retinóides incluem sequidão da pele e de mucosas, sangramento nasal ocasional, irritação e ressecamento ocular, prurido e descamação excessiva, que pode ser justificado pelo afinamento do estrato córneo. Os distúrbios psiquiátricos, quando retratados, aparecem como reações muito raras, o que por si só já evidencia sua inferior relevância na interferência da vida dos pacientes (Brito, Galindo, Santos, Sant'ana \& Rosendo, 2010).

Há claramente prejuízos físicos, sociológicos e psicológicos causados pela doença. Da mesma forma, inúmeros são os casos de resultados positivos e satisfatórios ao final, ou no processo, do tratamento com o uso da Isotretinoína Oral, principalmente referindo-se ao estado mental dos pacientes com acne grave à moderada (Silva et al., 2009).

Dentre as razões para a escolha deste tema destaca-se a importância de se investigar e insistir na opção de tratar esses pacientes com o "Roacutan", a fim de que melhorem sua socialização com os demais e principalmente, sua autoestima, evidenciando os efeitos benéficos trazidos pelo tratamento e desmistificando assim que o medicamento seja um potencializador de distúrbios depressivos, até que existam casos o suficiente de tais reações para que se prove o contrário. Desta forma, o objetivo desse estudo é analisar, a partir de um acompanhamento prospectivo, o impacto na saúde mental dos pacientes com acne moderada e grave que fazem uso da Isotretinoína.

\section{Metodologia}

Trata-se um estudo qualitativo de caráter descritivo, definido por Creswell (2009), como aquele que utiliza estratégias investigativas, além de métodos de coleta de dados, análise e a interpretação dos mesmos. A pesquisa aqui referida, foi realizada em um município da Paraíba e encaminhada anteriormente para análise do Comitê de Ética do Centro Universitário de Patos - UNIFIP, tendo obtido aprovação sob CAAE nº 39871220.3.0000.5181

Os participantes da pesquisa foram escolhidos de acordo com o fluxo de funcionamento no centro de referência, no período de março de 2021, no qual, preenchessem os critérios de inclusão: pacientes adolescentes tardios e adultos jovens, sem distinção de sexo, acometidos com a acne de grau moderado à grave em uso da Isotretinoína Oral. Todos eles consentiram suas entrevistas, com autorização por escrito mediante assinatura do Termo de Consentimento Livre e Esclarecido (TCLE). Com isso, foram obtidos um total de 10 pacientes, no período de um mês.

Para coleta de dados, foram produzidas entrevistas individuais utilizando-se de um roteiro estruturado composto por sete perguntas norteadoras desenvolvidas pelos pesquisadores, baseadas em: quais sentimentos os pacientes tinham durante o tratamento e antes dele; como, e se, o medicamento interferiu na vida social diária e nas relações interpessoais; o nível de satisfação dos entrevistados com a aparência de sua pele; e a possível ocorrência de pensamentos negativos ou autodepreciativos frente ao tratamento com a Isotretinoína. As entrevistas foram realizadas durante consulta, com duração 
média de 25 minutos, sendo criptografadas, gravadas e transcritas na íntegra. Os entrevistados são identificados pela letra P, e o número que acompanha tal consoante está de acordo com a ordem das entrevistas realizadas.

Ao tomar Bardin (2011) como referência, todas as respostas obtidas, tiveram avaliação pela análise de conteúdo. Esse método é formado por etapas, sendo elas: Pré-Análise, Exploração do Material e Interpretação dos resultados obtidos, buscando assim agregar um melhor embasamento à interpretação e seguimento ao estudo.

\section{Resultados e Discussão}

Quanto a caracterização das variáveis sociodemográficas obtidas, observou-se que os 10 participantes em sua maioria eram solteiros, estudantes, e variando entre faixas etárias de adolescentes tardios a adultos jovens.

A seguir são abordadas categorias selecionadas de acordo com as respostas dos entrevistados. Essas foram identificadas, agrupando-se as respostas mais pertinentes, relevantes e significativas dos pacientes. Serão apresentados trechos dos depoimentos com sua respectiva autoria.

\subsection{Insatisfação anterior ao tratamento}

Os pacientes entrevistados, em sua grande maioria, relataram sentimentos negativos principalmente de frustração, vergonha e desconforto, na fase da doença anterior ao início do tratamento. Percebe-se um padrão de tamanho desgosto e incômodo com a interferência da acne e suas manifestações na vida dos pacientes, no auge de sua juventude. O fato de já terem procurado por tratamentos anteriormente e todos terem apresentado ineficácia, os frusta ainda mais.

Nota-se que somente após serem submetidos a peelings químicos, limpezas de pele e administração dos mais diversos produtos, é que eles são devidamente orientados ao uso da Isotretinoína, quando muitas vezes, já estão com problemas psicológicos relacionados à grande queda de sua autoestima e desprazer com uma pele bastante desgastada, a depender do grau da doença.

\section{P1: Eu me sentia frustrada.}

P3: Antes de começar o tratamento, como tava muita acne né, eu só andava de maquiagem e não gostava de me olhar no espelho[...]

P4: Muito envergonhada, porquê foi uma coisa que surgiu muito de repente e aí com o passar do tempo não tinha mais como esconder.

P5: Eu evitava sair de casa principalmente pela questão estética que me deixava muito desconfortável. [...] $O$ tratamento na verdade ajudou a me sentir segura com minha questão estética e assim ter mais convívio social.

P6: Muito. Na verdade, muitas vezes evitava até sair de casa, com auto estima baixa, e até mesmo quando alguém fazia algum elogio quanto a minha aparência, ficava achando que era para me agradar.

P8: Em alguns momentos sim, muitas vezes eu parava pra pensar que quase nenhum dos meus/minhas amigos/amigas tinham acne, e o quanto aquilo de deixava mais feio.

P9: Sim. Tava tendo acne na região do colo e nas costas, isso inclusive me fez parar de querer usar roupa q mostrasse (roupas que gosto.)

P10: Envergonhada, com certeza! As espinhas me incomodavam e eu não conseguia escondê-las com maquiagem. 
No Grau III, a Acne é do tipo Nodulocística, apresentando comedões abertos, seborréia, pápulas, pústulas e a formação de nódulos furunculóides. A partir daí, inicia-se o aparecimento de cicatrizes. O Grau IV, identificado pela Acne Conglobata, com maior índice de acometimento no sexo masculino, é composto pela soma de cistos e nódulos com pus, volumosos e em grande quantidade, com maior predominância em face, pescoço e tórax (Belda, Di Chiacchio \& Criado, 2018)

Alguns autores, ainda, consideram a classificação de um Grau V para um quadro mais raro e complexo da doença, denominado de Acne Fulminans. Para tal, há a junção das características até então apresentadas nos casos mais leves, com alterações sistêmicas, tais como: lesões úlcero-hemorrágicas, poliartralgia, leucocitose e febre. Esse tipo de acne é muito mais comum em homens jovens (Belda, Di Chiacchio \& Criado, 2018).

Analisa-se que a acne vulgar é uma condição patológica que interfere não só de forma sensorial, ao causar dor e incômodo, como também no emocional do indivíduo acometido, ao adentrar na sua estética. Há claramente prejuízos físicos, sociológicos e psicológicos causados pela doença (Silva et al., 2009).

\subsection{Interferência do tratamento na vida social}

Os participantes da entrevista relataram influência do tratamento majoritariamente positiva e direta em sua vivência social, quando desconsiderados os efeitos físicos colaterais da medicação, descrevendo-a com as sensações de alívio, conforto, bem-estar e satisfação. As únicas queixas observadas pelas descrições dos pacientes são claramente relacionadas aos efeitos adversos já esperados pelo medicamento, e em sua maioria manifestam-se apenas fisicamente, por meio de uma maior sensibilidade da pele e sendo possível melhorá-las com a simples utilização, por exemplo, de protetores e hidratantes (geralmente faciais e labiais). Com a positividade da interferência do tratamento na vida dos pacientes e as sensações por eles citadas, nota-se um convívio em sociedade mais fácil ao se adquirir novamente autoconfiança e, progressivamente, o retorno de sua autoestima.

\section{P1: Hoje eu sinto que minha pele ficou maravilhosa!}

P2: Eu espero que a minha acne só regrida, que não fiquem cicatrizes, uma má aparência[...]

P3: Não, porque apesar de ainda estar inflamado porque comecei há 2 meses, eu não to sentindo o incômodo que eu sentia antes, em outro tratamento.

P4: No começo interferiu muito, principalmente na minha vida social. Sim, porque foi no auge que meu rosto começou a estourar [...]

P5: No início foi um processo de aceitação, devido ao ressecamento e ter que me adaptar a maiores cuidados para com a pele. Porém, ao decorrer do tratamento, ver minha pele "limpa" me trouxe uma sensação de segurança, de conforto e bem-estar. Conforme foi evoluindo o tratamento eu fui percebendo que realmente valeu a pena. Me senti muito bem quanto ao resultado nos últimos meses que foram ficando evidentes de fato.

P6: Acho, passei a me sentir melhor, até mais seguro de certa forma, quando comecei a me ver diferente. Vendo a melhora gradual e significativa me fez ter uma vida social de fato, sair com amigos, conhecer novas pessoas e lugares, parece que tudo era novo, sensação de recomeço. No último mês eu estava me sentindo super bem, a minha aparência já era outra, então eu não tinha problema algum em sair e ter vida social, ao contrário, comecei a sair bem mais, fazer coisas que antes evitava por não gostar da aparência. Tive a sensação de recomeço, tinha ficado 
para trás aquela pessoa que se sentia insegura, que evitava ficar muito próximo dos outros, por pensar que seria julgado pela aparência, ali havia mudado não só uma aparência, mas uma pessoa por inteira.

P7: Não, pelo o contrário melhorou meu convívio. Porque eu sei que eu vou melhorar mais do que ficava desconfortável.

P8: Não, não! O Roacutan não me impede de fazer praticamente nada, tem suas restrições, mas nada que chegue a impedir a minha vida social diária. Até porque por incrível que pareça, o Roacutan não "estragou" o meu rosto e nem me deu tantas reações como eu temia. Sempre, sempre meu rosto foi bem cheio de espinhas, quando comecei o tratamento, temia os tantos efeitos do medicamento e que a minha pele ficasse ainda mais horrível, mas recebi surpresas bem positivas, minha pele encheu um pouco mais no Início, mas depois a quantidade só diminui, então eu descreveria SATISFAÇÃO.

P9: No último mês (em tratamento) pele já melhorou bastante. Quase 100\%. Então diria que senti satisfação na aparência da minha pele.

O tratamento com a isotretinoína reduz significativamente as lesões da acne, porém efeitos adversos psiquiátricos podem ser comuns, tais como rebaixamento do humor, fadiga, alucinações, insônia e letargia (Li et al., 2019). Diferentes sistemas podem ser afetados, tais como o sanguíneo com aumento da concentração sérica de lipídios (hipertrigliceridemia) e elevação das enzimas hepáticas (ALT e AST); o dermatológico, sendo as manifestações mais comuns no tratamento com isotretinoína oral, com queilite e xerose sendo os mais comuns. Em última instância, o efeito adverso dermatológico mais grave é a síndrome de Stevens-Johnson, que afeta a pele e as membranas mucosas, sendo classificada como uma emergência médica e que requer suspensão do tratamento e internação para controle de sintomas e complicações (Vallerand et al., 2018).

Ouvidos, nariz e garganta também podem ser afetados com ressecamento das suas membranas mucosas. Problemas gastrointestinais são menos comuns, mas podem ocorrer e os mais registrados são náuseas, vômitos e redução do apetite. Sintomas oculares são frequentes e os mais comuns são ressecamento dos olhos, irritação e conjuntivite. Dentre os sintomas psiquiátricos ou psicossomáticos mais comumente relatados, destacam-se letargia e fadiga. Outros sintomas como rebaixamento do humor, insônia e alucinações também foram atribuídos ao uso da isotretinoína oral (Vallerand et al., 2018).

\subsection{Prognóstico para/com o tratamento}

Os entrevistados, em sua totalidade, descreveram suas experiências com a Isotretinoína como um resultado positivo e fortemente eficaz, superando até mesmo suas expectativas com o medicamento. Muitos deles, anteriormente apreensivos tanto com os boatos errôneos a respeito do tratamento medicamentoso, como com a situação de sua acne, relataram a eficácia da Isotretinoína na solução do seu problema e ainda a esperança de que ao finalizarem a terapêutica, não haja retorno algum dos sintomas estéticos que tanto o incomodavam e nem a prevalência das tão temidas cicatrizes.

P1: Penso que foi muito efetivo no meu caso. Passei por uma série de tentativas com outros medicamentos antes, mas sem sucesso, só me frustrava. O único eficaz foi a isotretinoína.

P2: Vejo mais como um aspecto positivo, porque por mais que a gente saiba que a isotretinoína cause efeitos colaterais, como o ressecamento dos lábios que é praticamente inevitável e incomoda, mas na minha concepção as vantagens ao final do tratamento são superiores às desvantagens, então eu acho sim que o resultado do Roacutan é positivo. 
P3: Na segunda semana eu já comecei a sentir a pele bem ressecada e seca e nascendo algumas espinhas mas ao mesmo tempo secando outras, aí tem dias que eu acho ela ótima, já em outras nasce alguma espinha e assim vai, mas eu estou achando ela ótima, apesar de muito ressecada. Até agora eu estou achando bem positivo, apesar dos efeitos colaterais que eu sei que tem inúmeros, acho que eu estou bem isenta deles, só sinto mesmo o ressecamento da pele e ela bem sensivel

P4: Evolução maravilhosa. Positiva nível máximo!

P5: Como um aspecto positivo, com certeza, principalmente porquê antes de iniciar o tratamento com Roacutan, eu passei por outros tratamentos tópicos e que não tinham um resultado efetivo. Mas com a busca do profissional correto, que me tirou dúvidas e medo acerca do roacutan, tive um resultado incrivel ao qual eu super indicaria o uso.

P6: Nos primeiros dias percebi uma piora, mas nada muito significativa, o que houve de fato foi uma melhora gradual, a oleosidade reduzindo a cada dia, as acnes cicatrizando cada vez mais até as espinhas ficarem esporádicas, sentindo uma pele mais firme. Extremamente positivo. Acho que foi a melhor conduta médica tomada e a melhor decisão que fiz. Tudo que nos renova, vem para somar em todos os aspectos, e no meu caso que praticamente não tive efeitos colaterais, a medicação de fato só veio me trazer benefícios.

P8: É normal durante o tratamento apresentar ainda acnes, mesmo que poucas. Foi uma evolução enorme que me enche de orgulho, felicidade e satisfação. POSITIVO, como eu poderia considerar algo que me fez tão bem como algo negativo?

P9: Positivo. Em mim não houve grandes efeitos colaterais [...]

P10: Positivo demais! Sem contar que antes mesmo de começar a usar pesquisei e vi relatos que quem usa, dificilmente volta a ter espinhas no futuro. É o que espero pra mim!

Popularmente denominado de "Roacutan", trata-se de um medicamento aprovado pelo FDA para tratar acne grave. Sua eficácia atua também em casos de recorrência da acne, resistência a outros tipos de tratamento, e ainda, nos quadros de acometimento moderado, em que se utilizam doses mais baixas $(0,3 \mathrm{mg} / \mathrm{kg} / \mathrm{dia})$, produzindo menos efeitos adversos dessa forma (Titus \& Hodge, 2012). Na grande maioria dos casos, o tratamento é feito com 0,6 a $0,75 \mathrm{mg} / \mathrm{Kg} / \mathrm{dia}$ de isotretinoína por 16 a 35 semanas, garantindo bons resultados após esse período (Silva et al., 2009).

O composto atua especificamente interferindo na atividade das glândulas sebáceas, suprimindo-as. Dessa forma, agirá efetivamente na lesão primária da acne, o microcomedão, e, através de suas propriedades anti-inflamatórias e imunomoduladoras, reduz o quadro inflamatório instalado e a proliferação da doença (BRITO et al., 2010). Em outras palavras, o Ácido Retinóico se liga a receptores para-retinóides presentes nas glândulas sebáceas, reduzindo assim a sua atividade, o total de sebo que a mesma irá produzir e o seu volume. Além disso, há a redução da formação de microcomedões devido à normalização da queratinização folicular, o que contribui diretamente em uma menor proliferação bacteriana. Tudo isso ocorre geralmente após quatro semanas de tratamento (Belda, Di chiacchio \& Criado, 2018).

\subsection{Intervenção do processo de tratamento na saúde mental}

Os pacientes que participaram das entrevistas, abordaram, ou não sentir efeitos psíquicos negativos e prejuízo emocional em sua rotina, ou relataram sentimentos de ansiedade quanto aos resultados do tratamento e leve preocupação 
referente aos efeitos colaterais que o medicamento proporciona. É válido ressaltar que, alguns problemas psicológicos relatados foram claramente atribuídos às expectativas de resultados positivos a curto prazo, definidos assim como "ansiedade", e alguns ainda, tiveram sua origem anterior ao início do uso da Isotretinoína Oral, em outras situações de vida, podendo assim ter se prolongado ao período de terapêutica. Todos estes fatores acabam por fomentar uma imagem erroneamente negativa de que o medicamento é um "monstro" para a saúde mental dos pacientes, quando na verdade, sabe-se que não há outro vilão nesse processo que não seja a acne.

P1: Eu interrompi o primeiro tratamento porque tive crise de ansiedade e o psiquiatra mandou parar, mas meu médico dermatologista disse que não tinha indicação, inclusive retornei ao tratamento.

P2: No meu primeiro tratamento eu tive algumas crises de ansiedade, mas no meu segundo tratamento isso não aconteceu $[\ldots]$

P3: Como eu ainda estou no início de tratamento, me incomoda porque a pele ta ressecada, inflamada, mas eu já sinto um alívio muito grande porque eu sei que a tendência é melhorar cada vez mais.

P4: Eu senti um estresse absurdo, como se nada agradasse. Foi um misto de medo (pelas reações) e alívio (por saber que era só uma fase e logo ia passar).

P5: Não tive esse tipo de sentimentolefeito negativo após iniciar o tratamento.

P6: Não, nunca tive esses pensamentos, sei que o Roacutan traz consigo inúmeros efeitos colaterais, mas tive apenas pensamentos bons e gratificantes.

P8: Outra coisa que temia muito, mas não, não! Foram poucas as vezes que isso aconteceu.

P9: Não. Nada, roacutan não mudou em nada no meu emocional.

P10: Venho percebendo uma melhora significativa. Espinhas menos inflamadas e elevadas, com aspecto secante e clareador. Sensação eficaz essa que não percebi em vários tratamentos que testei anteriormente. Apesar de perceber que durante o início do tratamento as acnes aumentaram, saíram todas de uma vez, não me incomodava, pois, foi me avisado com antecedência e eu sabia que a melhora estava próxima.

Em uma meta-análise de 31 estudos realizada por Huang, Yu-Chen e Cheng (2017) não foi identificado associação direta entre uso de isotretinoína e depressão. Na verdade, foi observado que os sintomas depressivos melhoraram com a efetivação e seguimento do tratamento, coincidindo com a melhora das lesões da acne. Dessa forma, o tratamento com isotretinoína oral não esteve relacionado com o desenvolvimento de sintomas depressivos nos estudos realizados por esta metaanálise.

Nesse mesmo estudo, a associação entre sintomas depressivos e acne identificada esteve relacionada com os efeitos adversos do tratamento, maior duração da acne bem como a severidade das lesões e o estado mental dos pacientes no início do tratamento, quando as lesões estavam mais visíveis (Schmitt \& Luvizotto, 2020).

É importante identificar a associação entre a intensidade dos efeitos adversos muco cutâneos e os sintomas depressivos nos pacientes em tratamento com a isotretinoína, aplicando-se medidas que efetivamente reduzam os sintomas e orientando o paciente sobre a eventual possibilidade de manifestações clínicas no início do tratamento (Schmitt \& Luvizotto, 2020). 


\section{Conclusão}

A acne é uma condição cutânea que envolve fatores genéticos e hormonais e pode ter um impacto psicológico e social bastante significativo em um indivíduo. Os sentimentos que interpassam o portador de acne são em geral bastante negativos, como insegurança, baixa autoestima, introspecção, além de restrições cotidianas. A saúde mental dos pacientes que possuem acne grave é por si só abalada, devido aos efeitos carreadores da doença. Essa é a grande vilã que prejudica além da pele, o psicológico dos acometidos, ocasionando aos mesmos grandes problemas de imagem.

A Isotretinoína oral, composto eficaz como uma opção de tratamento aos casos de acne grave a moderada, proporciona um cenário de diversos efeitos colaterais que provocam um certo temor aos que não conhecem. No entanto, é importante que os médicos, além de orientarem o devido uso e suas consequências, conscientizem os pacientes de que haverá, em sua grande maioria, o resultado positivo tão almejado do medicamento, principalmente levando-se em consideração a promoção do seu bem-estar.

Finalmente, esses elementos devem ser levados em consideração no prognóstico e tratamento dos pacientes portadores de acne grave a moderada, com a finalidade de resultados mais satisfatórios. Além disso, os médicos desmistificam assim o temor popular de uma associação negativa a um medicamento de potencial eficácia.

O estudo realizado contou com limitações quanto ao número reduzido de pacientes do grupo amostral devido ao pouco tempo de pesquisa. Logo, para uma análise ainda mais a fundo, seria necessário um período maior de avaliações. Ademais, estudos futuros que evidenciem a busca por terapia como um adjuvante eficaz a portadores de doenças dermatológicas, é uma sugestão pertinente para o tema.

\section{Referências}

Abreu, E. C. M., Martins, A. B. T, Uchôa, A. K. A, Falcão, C. de. S. V., \& Bezerra, L. M. M. (2013). Conhecimento de alunos da rede pública do município de Canindé/CE sobre o tratamento de acne vulgar. Rev.Fisioter. S. Fun., Fortaleza, 2(1), 28-34.

Araújo, A.P.S., Delgado, D.C., \& Marçal R. (2011). Acne diferentes tipologias e formas de tratamento. VII Encontro Internacional de Produção Científica. http://www.cesumar.br/prppge/pesquisa/epcc2011/or2.php

Azulay, R.D. (2013). Dermatologia. 6. ed. Rio de Janeiro:Guanabara Koogan.

Barbosa, G. S. L., Costa, C. P. M., Borges, M. V. R., Attem, M. S., Cacau, B. L., Lopes, M. S., Mass, D. W., Pereira, B. S., Coutinho, M. Ângela de O. C., Luz, F. A., Santos, K. R. dos., \& Fontenelle, L. F. V. (2021). Tratamentos medicamentosos para acne vulgar em adolescentes e adultos jovens. Pesquisa, Sociedade e Desenvolvimento, 10 (5), e39010515094. https://doi.org/10.33448/rsd-v10i5.15094

Belda, W. Jr, Di chiacchio N., \& Criado, P. R. (2018). Tratado de Dermatologia. $3^{\text {a } . ~ e d i c ̧ a ̃ o . ~ S a ̃ o ~ P a u l o: ~ E d . A t h e n e u . ~}$

Brito, F. M. M., Galindo, J. C.S., Santos, J. B., Sant'ana, I. P., \& Rosendo, L. H. P. M. (2010). Avaliação dos efeitos adversos clínicos e alterações laboratoriais em pacientes com acne vulgar tratados com isotretinoína oral. Anais Brasileiros de Dermatologia, 85(3), 331-337.

Creswell, J. (2009). Research design:qualitative, quantitative, and mixed methods approaches. Thousand Oaks: Sage.

Costa, F. V., Souza, L. S. de, Conradesque, P. R., Carniel, P., \& Zago, Â. (2020). Indução percutânea de colágeno como tratamento de cicatrizes Acne vulgaris: uma revisão integrativa. Research, Society and Development, 9 (8), e252985706. https://doi.org/10.33448/rsd-v9i8.5706

Cubas, V. M. \& Ribas, J. L. C. (2021). Fitoterapia alternativa para o tratamento da acne. Pesquisa, Sociedade e Desenvolvimento, 10 (2), e12810212153. https://doi.org/10.33448/rsd-v10i2.12153

Huang, Y. C., \& Cheng, Y. C. (2017). Isotretinoin treatment for acne and risk of depression: A systematic review and meta-analysis. Journal of the American Academy of Dermatology, 76(6), 1068-1076.e9. https://doi.org/10.1016/j.jaad.2016.12.028

Isidorio, E. C., Guido, J. V. G., Sena, B. L. T., Oliveira, A. S. de., Santos, R. M. S. M. dos, Camargo, C. A. C. M., \& Camargo, M.A.F. (2020). O uso do tratamento psicológico para melhorar a qualidade de vida de pacientes dermatológicos: uma revisão integrativa da literatura. Research, Society and Development , 9 (10), e7699109191. https://doi.org/10.33448/rsd-v9i10.9191

Lima, M. F. da silva, Barros, V. J. da silva, \& Neto, M. P. L. (2020). Analysis of oral isotretinoin consumption in the specialized component of pharmaceutical assistance in the state of Piauí. Research, Society and Development, 9(2), e170922235. https://doi.org/10.33448/rsd-v9i2.2235

Li, C., Chen, J., Wang, W., Ai, M., Zhang, Q., \& Kuang, L. (2019). Use of isotretinoin and risk of depression in patients with acne: a systematic review and meta-analysis. BMJ open, 9(1), e021549. https://doi.org/10.1136/bmjopen-2018-021549 
Research, Society and Development, v. 10, n. 9, e14710917770, 2021

(CC BY 4.0) | ISSN 2525-3409 | DOI: http://dx.doi.org/10.33448/rsd-v10i9.17770

Miranda, C. C. da Silva. (2020). Atividade antibiótica de extratos e óleos essenciais contra Propionibacterium acnes. Research, Society and Development , 9 (10), e2569108070. https://doi.org/10.33448/rsd-v9i10.8070

Sampaio, S. A. P., \& Bagatin, E. (2008). Experiência de 65 anos no tratamento da acne e de 26 anos com isotretinoína oral. Anais Brasileiros de Dermatologia, 83(4), 361-367.

Sampaio, S., \& Rivitti, E.A., (2014). Manual de Dermatologia Clínica de Sampaio e Rivitti. Artes Médicas.

Silva, E. D. da.Jr, Sette, I. M. F., Belém, L. de. F., Janebro, D. I., Pereira, G. J. da. S., Barbosa, J. A. A. B., \& Menezes, M. D. de. S. F. (2009). Isotretinoína no tratamento da acne: riscos x benefícios. Revista Brasileira de Farmacologia, Paraíba, 90(3), 186-189.

Schmitt, V. J., \& Luvizotto, P.P. (2020). Sintomas depressivos antes e durante o tratamento da acne com isotretinoína e suas correlações: um estudo prospectivo. Anais Brasileiros de Dermatologia, 95(6), 760-763.

Titus, S., \& Hodge, J. (2012). Diagnosis and treatment of acne. American family physician, 86(8), 734-740.

Vallerand, I. A., Lewinson, R. T., Farris, M. S., Sibley, C. D., Ramien, M. L., Bulloch, A. G. M., \& Patten, S. B. (2018). Eficácia e eventos adversos da isotretinoína oral para acne: uma revisão sistemática. British Journal of Dermatology, 178(1), 76-85.

Vinhal, D. C., Roberth, A. O., Ortence, V. O. P., \& Diniz, D. G. A. (2014) Terapia retinóide na acne vulgar. Revista Eletrônica de Farmácia, $11(3), 22$. 